ANNALES

POLONICI MATHEMATICI

$87(2005)$

\title{
On gradient at infinity of semialgebraic functions
}

\author{
by Didier D'Acunto (Pisa) and Vincent Grandjean (Bath)
}

\begin{abstract}
Let $f: \mathbb{R}^{n} \rightarrow \mathbb{R}$ be a $C^{2}$ semialgebraic function and let $c$ be an asymptotic critical value of $f$. We prove that there exists a smallest rational number $\varrho_{c} \leq 1$ such that $|x| \cdot|\nabla f|$ and $|f(x)-c|^{\varrho_{c}}$ are separated at infinity. If $c$ is a regular value and $\varrho_{c}<1$, then $f$ is a locally trivial fibration over $c$, and the trivialisation is realised by the flow of the gradient field of $f$.
\end{abstract}

1. Introduction. As a consequence of the fundamental paper of Thom (cf. [Th]) about conditions ensuring the local topological triviality of a smooth mapping, given a polynomial $f: \mathbb{C}^{n} \rightarrow \mathbb{C}$, there exists a finite subset of values $\Lambda \subset \mathbb{C}$ such that the function $f$ induces a locally trivial fibration from $\mathbb{C}^{n} \backslash f^{-1}(\Lambda)$ onto $\mathbb{C} \backslash \Lambda$. The smallest such subset of $\mathbb{C}$, denoted by $B(f)$, is called the set of bifurcation values of the function $f$. It contains the usual critical values of $f$. Unfortunately, there may exist regular values that are also bifurcation values. But Thom did not give any way to find these regular bifurcation values.

A few years later, Pham, in relation to convergence of oscillating integrals, exhibited a condition ensuring that a complex polynomial $f$ trivialises over a neighbourhood of a regular value $c \in \mathbb{C}$ : the Malgrange condition (cf. [Ph]). Roughly speaking, this condition means that the norm of the gradient is not too small in a neighbourhood of the germ at infinity of the given level $f^{-1}(c)$.

The set of values at which the Malgrange condition is not satisfied is actually finite (see [Ti1]). Moreover the Malgrange condition fails at any bifurcation value that is also regular. Finally, Parusiński proved that for a complex polynomial with isolated singularities at infinity, any regular value

2000 Mathematics Subject Classification: Primary 32Bxx, 34Cxx; Secondary 32Sxx, $14 \mathrm{P} 10$.

Key words and phrases: semialgebraic functions, gradient trajectories, Łojasiewicz inequalities, Malgrange condition.

Supported by the European research network IHP-RAAG contract number HPRNCT-2001-00271 
at which the Malgrange condition fails is a bifurcation value (see $[\mathrm{Pa}]$ ). Yet, in full generality, we still do not know if this property is true for any complex polynomial.

Now, let us turn to the case of a real polynomial $f$. As in the complex situation, the set of bifurcation values, as defined above, is finite, as also is the set of values at which the Malgrange condition is not satisfied (see [Ve], [Ti1]). Again, no regular bifurcation value satisfies the Malgrange condition. As in the complex case, this hopefully ensures a fibration theorem outside these special fibres and the critical fibres. But in the real case, the result of Parusiński is no longer true. A regular value of a real plane polynomial at which the Malgrange condition fails is not necessarily a bifurcation value (see the King-Tibăr-Zaharia and Parusiński examples in Section 5).

When the Malgrange condition is satisfied at a regular value $c$, the function is locally trivial over a neighbourhood of $c$. Moreover, this trivialisation can be realised by the flow of the gradient vector field $\nabla f$.

At the early stage of this work, we expected that, at least in the real plane case, trivialising by $\nabla f$ in a neighbourhood of a regular value $c$ and having the Malgrange condition satisfied at $c$ were equivalent conditions. But this belief was erroneous, as shown by the Parusiński example in Section 5 .

Nevertheless, these examples have led us to try to understand more closely the connections between the behaviour of the trajectories of the gradient field $\nabla f$, the asymptotic geometry of the neighbouring levels of the level $c$ and the failure of the Malgrange condition at $c$. We have been particularly interested in the trajectories leaving any compact subset of $\mathbb{R}^{n}$ and along which $f$ tends to a finite value $c$ at infinity. We will not explore here the very difficult problem of the qualitative behaviour of such trajectories, but they have led us to the discovery of the Kurdyka-Lojasiewicz exponent at infinity for $c$ and its corresponding gradient-like inequality in a neighbourhood of the level $c$ at infinity, a notion that actually improves the Malgrange condition considerably, and with a geometric content closely connected to the foliation by the levels of $f$.

In this article we will work with $C^{1}$ (or $C^{2}$ depending on the context) semialgebraic functions, since most of the results we are interested in, originally stated in the polynomial case, are also available in the semialgebraic frame.

Conventions. Let $u$ and $v$ be two germs at infinity of single real variable functions. We write $u \sim v$ to mean that the ratio $u / v$ has a non-zero finite limit at infinity. We write $u \simeq v$ when the limit of $u / v$ at infinity is 1 .

2. Asymptotic critical values and the embedding theorem. Let $f: \mathbb{R}^{n} \rightarrow \mathbb{R}$ be a $C^{1}$ semialgebraic function. Just as in the introduction, 
the fibres of $f$ exhibit only finitely many topological types ([Ve] or [KOS]). The values at which the topology changes are called bifurcation values (or atypical values) of $f$. Any other value is called a typical value. The set of atypical values is finite and denoted by $B(f)$. In this set, we distinguish two sorts of values: the usual critical values, denoted by $K_{0}(f)$, and $K_{\infty}(f)$, the asymptotic critical values, at which the Malgrange condition fails:

Definition 2.1. The function $f$ satisfies the Malgrange condition (M) at a value $t \in \mathbb{R}$ if there exists a constant $C>0$ such that for sufficiently large $x$ and $f(x)$ sufficiently close to $t$ the following inequality holds:

$$
|x| \cdot|\nabla f(x)| \geq C \text {. }
$$

Equivalently, $c \in K_{\infty}(f)$ if there exists an unbounded sequence $\left\{x_{\nu}\right\}_{\nu}$ $\in \mathbb{R}^{n}$ such that $f\left(x_{\nu}\right) \rightarrow c$ and $\left|x_{\nu}\right| \cdot\left|\nabla f\left(x_{\nu}\right)\right| \rightarrow 0$.

REMARK 2.2. The previous definition and the notion of critical values at infinity also make sense for any $C^{1}$ real function defined on an unbounded open subset of $\mathbb{R}^{n}$, as well as for complex polynomials.

Let $K(f)=K_{0}(f) \cup K_{\infty}(f)$ be the set of generalised critical values.

In the real case, condition (M) ensures the trivialisation via the gradient field $\nabla f$. To be more precise, assume that $f$ denotes a $C^{2}$ semialgebraic function. Let $\Phi$ be the local flow of $\nabla f /|\nabla f|^{2}$ defined as the mapping satisfying the following conditions:

$$
\frac{d \Phi}{d t}(x, t)=\frac{\nabla f}{|\nabla f|^{2}} \circ \Phi(x, t) \quad \text { and } \quad \Phi(x, 0)=x .
$$

Let us begin by stating an embedding theorem, which is fundamental to this work. Let $c$ be a regular value of $f$. Let $t$ be any regular value such that $[t, c[\cap K(f)=\emptyset$ if $t<c$, or $] c, t] \cap K(f)=\emptyset$ if $t>c$. Then we have:

ThEOREM 2.3 ([D'A2]). There exists a $C^{1}$ injective open immersion $\phi: f^{-1}(c) \rightarrow f^{-1}(t)$. More precisely, the flow of $\nabla f /|\nabla f|^{2}$ embeds each connected component of $f^{-1}(c)$ into a connected component of $f^{-1}(t)$.

REMARK 2.4. The mapping $\phi$ is in fact the restriction to $f^{-1}(c) \times\{t\}$ of the mapping $\Phi$. Such an embedding $\phi$ maps diffeomorphically the compact connected components of $f^{-1}(c)$ onto those of $f^{-1}(t)$.

If the flow of $\nabla f$, over a neighbourhood of a regular value $c$, does not trivialise $f$, then there is at least a trajectory of $\nabla f$ that never reaches the level $c$. More precisely, we introduce the following

Definition 2.5. An integral curve of $\nabla f$, leaving any compact subset of $\mathbb{R}^{n}$ and such that the function $f$ has a finite limit $c$ along a half-branch at infinity of this trajectory, is called an integral curve (or trajectory) of infinite length at $c$. 


\section{Kurdyka-Łojasiewicz exponent at infinity for an asymptotic} critical value. The standard Łojasiewicz gradient inequality states that if $f: U \rightarrow \mathbb{R}$ is an analytic function in a neighbourhood $U$ of the origin $0 \in \mathbb{R}^{n}$ such that $\nabla f(0)=0$, then there exist $U_{0} \subset U$ and positive numbers $\varrho$ and $C$ such that

$$
|\nabla f| \geq C|f-f(0)|^{\varrho} \quad \text { on } U_{0} .
$$

The infimum of the exponents $\varrho$ such that $|\nabla f||f-f(0)|^{-\varrho}$ has a positive limit along any sequence converging to 0 is called the Eojasiewicz exponent of $f$ and is a rational number lying in $] 0,1[$.

REMARK 3.1. Let $f: \mathbb{C}^{n} \rightarrow \mathbb{C}$ be a polynomial. There is already a notion of Łojasiewicz exponent at infinity, meaningful in this setting (see [Ha]). Namely, if $c$ is a regular value of $f$, the Lojasiewicz exponent at infinity for $c$ is the supremum of the real numbers $\theta$ for which there exists $C>0$ such that for all $x$ with $|x| \gg 1$ and $|f(x)-c| \ll 1$,

$$
|\nabla f(x)| \geq C|x|^{\theta} \text {. }
$$

Let $L_{c}(f)$ be this supremum. It is again a rational number and $c$ is an asymptotic critical value if and only if $L_{c}(f)<-1$.

Our purpose is to compare $|\nabla f(x)|$ with $|f(x)-c|$ for an asymptotic critical value $c$ of a semialgebraic function. The following result provides an analog at infinity of the standard Łojasiewicz gradient inequality stated above. This is the first important result of this article; to the best of our knowledge, it has not been known before.

Proposition 3.2. Let $f$ be a $C^{1}$ semialgebraic function. If $c \in \overline{\operatorname{Im} f}$, then there exist real numbers $C, R, \tau>0$ and a smallest rational number $\varrho_{c} \leq 1$ such that for all $x \in \mathbb{R}^{n}$ with $|x|>R$ and $|f(x)-c|<\tau$, we have

$$
|x| \cdot|\nabla f(x)| \geq C|f(x)-c|^{\varrho_{c}} .
$$

Proof. By the curve selection lemma, it suffices to prove this fact on semialgebraic curves having a half-branch at infinity. For simplicity we will only consider values $t<c$. Let $G$ be a semialgebraic half-branch at infinity, along which $f$ tends to $c \in \mathbb{R}$ at infinity. We can assume that $f$ is increasing along $G$. Let $\left[c-\tau, c\left[\ni t \mapsto g(t) \in \mathbb{R}^{n}\right.\right.$ be a semialgebraic parametrisation of the germ of $G$ at infinity satisfying $f \circ g(t)=t$ for each $t$. Then there exist a rational number $\eta>0$ and a positive real number $K$ such that

$$
|g(t)| \simeq K|t-c|^{-\eta} \quad \text { as } t \rightarrow c .
$$

By usual semialgebraic arguments, we get

$$
\left|g^{\prime}(t)\right| \simeq K \eta|t-c|^{-(1+\eta)} \quad \text { as } t \rightarrow c .
$$

Taking derivatives with respect to $t$, we obtain

$$
(f \circ g)^{\prime}(t)=\left\langle\nabla f(g(t)), g^{\prime}(t)\right\rangle=1 .
$$


Thus, we deduce

$$
|\nabla f(g(t))| \geq \frac{1}{2 K \eta}|t-c|^{\eta+1}
$$

and

$$
|g(t)| \cdot|\nabla f(g(t))| \geq \frac{1}{4 \eta}|t-c| .
$$

Since the function $t \mapsto f(g(t))$ is semialgebraic, there exists a rational number $\nu$ such that

$$
|g(t)| \cdot|\nabla f(g(t))| \sim|t-c|^{\nu} .
$$

From inequality (3.1) we obtain $\nu \leq 1$.

Let $\varrho_{c}$ be the infimum of these exponents $\nu$. Define

$$
E_{c}=\left\{q \in \mathbb{Q}: \lim _{|x| \rightarrow+\infty} \frac{|x| \cdot|\nabla f(x)|}{|f(x)-c|^{q}} \in \mathbb{R}_{+}^{*}, \lim _{|x| \rightarrow+\infty} f(x)=c\right\} .
$$

We easily verify that $E_{c}$ is a semialgebraic subset of $\mathbb{R}$ contained in $\mathbb{Q}$, hence it is finite (for details see [KMP, Proposition 4.2]). Thus $\varrho_{c}$ is rational.

Since there is yet a Łojasiewicz exponent at infinity (cf. Remark 3.1), we will refer to $\varrho_{c}$ as the Kurdyka-Eojasiewicz exponent at infinity of the function $f$ for the value $c$.

REMARK 3.3. Let us mention that Proposition 3.2 also holds when $f$ : $V \rightarrow L$ is a semialgebraic $C^{1}$ function, defined on a closed and connected semialgebraic $C^{1}$ submanifold $V$ of $\mathbb{R}^{n}$, equipped with the semialgebraic Riemannian metric induced from the Euclidean one.

The Malgrange condition corresponds to a value $c$ of the given function for which the Kurdyka-Eojasiewicz exponent at infinity for $c$ is less than or equal to 0 . The following proposition is just a rewriting of condition (M):

Proposition 3.4. Let $f$ be a $C^{1}$ semialgebraic function. Let $c \in \overline{\operatorname{Im} f}$. Then $c$ is an asymptotic critical value of $f$ if and only if the KurdykaEojasiewicz exponent at infinity of $f$ for $c$ is positive.

Let $c \in K_{\infty}(f) \backslash K_{0}(f)$ and let $\varrho_{c}$ be the Kurdyka-Lojasiewicz exponent at infinity for $c$. This number contains interesting information about the kind of value (typical or not) that $c$ could be, as shown by the following

THEOREM 3.5. Let $f$ be a $C^{2}$ semialgebraic function. If $\varrho_{c}<1$, then $f$ is a locally trivial fibration over c. Moreover, the fibration can be realised by the flow of $\nabla f /|\nabla f|^{2}$.

Proof. For simplicity we shall again only work with values $t<c$. Let $c_{0}<c$ be such that $\left[c_{0}, c\right] \cap K(f)=\{c\}$, and let $R, C>0$ be real numbers such that the assertion of Proposition 3.2 holds in $f^{-1}\left(\left[c_{0}, c[) \cap\{|x|>R\}\right.\right.$ with constant $C$. Let $x_{0} \in f^{-1}\left(c_{0}\right) \cap\{|x|>R\}$ and let $\gamma$ be a (maximal) 
trajectory of $\nabla f$ parametrised by the levels of $f$. So $\gamma$ satisfies the differential equation

$$
\gamma^{\prime}(t)=\mathbf{X}(\gamma(t)), \quad \gamma\left(c_{0}\right)=x_{0} \in f^{-1}\left(c_{0}\right),
$$

where $\mathbf{X}=\nabla f /|\nabla f|^{2}$. Thus, for each $t \in\left[c_{0}, c[\right.$, we obtain $f \circ \gamma(t)=t$.

Integrating (3.2) between $c_{0}$ and $t<c$, we obtain

$$
\int_{c_{0}}^{t} \gamma^{\prime}(s) d s=\int_{c_{0}}^{t} \mathbf{X}(\gamma(s)) d s .
$$

From (3.3), we get a first inequality

$$
|\gamma(t)| \leq\left|\gamma\left(c_{0}\right)\right|+\int_{c_{0}}^{t} \frac{d s}{|\nabla f(\gamma(s))|} .
$$

Using Proposition 3.2 we have

$$
|\gamma(t)| \leq\left|\gamma\left(c_{0}\right)\right|+\int_{c_{0}}^{t} \frac{|\gamma(s)|}{C|s-c| \varrho_{c}} d s .
$$

Then the Gronwall Lemma gives

$$
|\gamma(t)| \leq\left|\gamma\left(c_{0}\right)\right| \exp \int_{c_{0}}^{t} \frac{d s}{C|s-c| \varrho_{c}},
$$

which actually yields

$$
|\gamma(t)| \leq\left|\gamma\left(c_{0}\right)\right| \exp \frac{\left(c-c_{0}\right)^{1-\varrho_{c}}-(c-t)^{1-\varrho_{c}}}{C\left(1-\varrho_{c}\right)} .
$$

Hence $|\gamma(t)|$ has a finite limit as $t$ tends to $c$. This implies that the embedding $\phi$ of Theorem 2.3 is essentially a diffeomorphism from $f^{-1}(t)$ onto $f^{-1}(c)$. This ends the proof.

REMARK 3.6. Note that Theorem 3.5 also holds under the assumptions of Remark 3.3, provided the $C^{1}$ regularity of $f$ is replaced by $C^{2}$ regularity.

COROLlary 3.7. If $c$ is a regular value and a bifurcation value, then the Kurdyka-Lojasiewicz at infinity for $c$ is equal to 1.

Proof. Since we cannot trivialise the function $f$ over a neighbourhood of $c$, from Theorem 3.5, the exponent has to be 1 .

When $c$ belongs to $K_{\infty}(f) \backslash B(f)$, the function $f$ induces a locally trivial fibration over a neighbourhood of $c$. Moreover, this trivialisation is provided by the flow of $\nabla f /|\nabla f|^{2}$ when the Kurdyka--Lojasiewicz exponent at infinity for $c$ is strictly less than 1. From the view point of Definition 2.5, Theorem 3.5 can be stated in another way:

Corollary 3.8. Let $\Gamma$ be a trajectory of $\nabla f$ of infinite length at $c$. Then the Kurdyka-Lojasiewicz exponent at infinity of $c$ is equal to 1. 
4. Kurdyka-Kojasiewicz exponent of complex polynomials. Let $f: \mathbb{C}^{n} \rightarrow \mathbb{C}$ be a complex polynomial. As mentioned in Section 2, we can define the set $K_{\infty}(f)$ of asymptotic critical values, which is a finite subset of $\mathbb{C}$ ([Ti1]). Again, we write $K(f)=K_{0}(f) \cup K_{\infty}(f)$, the set of generalised critical values. If $t \in \mathbb{C} \backslash K(f)$ then $f$ is a locally trivial fibration over $t$ ([Ti1], [Ti2]).

There also exists an analog of the Embedding Theorem 2.3 in the complex case. Namely, if $c \in K_{\infty}(f) \backslash K_{0}(f)$ and $t \in \mathbb{C} \backslash K(f)$ then we have

THEOREM 4.1 ([D'A2]). There exists an embedding $\varphi_{c, t}: f^{-1}(c) \rightarrow$ $f^{-1}(t)$.

Let grad $f$ be the polynomial vector field in $\mathbb{C}^{n}$ whose components are $\left(\partial f(z) / \partial z_{1}, \ldots, \partial f(z) / \partial z_{1}\right)$, where $z=\left(z_{1}, \ldots, z_{n}\right)$ is a system of coordinates in $\mathbb{C}^{n}$. Denote by $\|w\|$ the norm of the complex vector $w \in \mathbb{C}^{n}$. The proof of Theorem 4.1 (see [D'A2] for details) combined with the proof of Proposition 3.2 gives

Proposition 4.2. There exist $C>0$ and a rational number $0<\varrho \leq 1$ such that for sufficiently large $\|z\|$ and sufficiently small $|f(z)-c|$, we have

$$
\|z\| \cdot\|\operatorname{grad} f(z)\| \geq C|f(z)-c|^{\varrho} .
$$

As before, the infimum of such exponents $\varrho$ is positive and rational. Again, we denote it by $\varrho_{c}$, and call the Kurdyka-Lojasiewicz exponent at infinity of $f$ for $c$.

The complex situation is much more rigid than the real one. When the function $f$ has only isolated singularities at infinity, knowing $\varrho_{c}$ decides whether the regular value $c$ is typical or atypical. Under this hypothesis, Parusiński proved that any asymptotic critical value is a bifurcation value $[\mathrm{Pa}]$, that is, $B(f)=K_{\infty}(f) \cup K_{0}(f)$.

THEOREM 4.3. Let $f$ be a complex polynomial with only isolated singularities at infinity. A regular value $c$ is a bifurcation value if and only if the Kurdyka-Lojasiewicz exponent at infinity $\varrho_{c}$ is equal to 1.

Proof. If $\varrho_{c}=1$, then $c$ is an asymptotic critical value, and by Parusiński's result $[\mathrm{Pa}]$, it is necessarily a bifurcation value.

Let $c$ be a regular bifurcation value. So the embedding $\varphi_{c, t}: f^{-1}(c) \rightarrow$ $f^{-1}(t)$ is not onto for any typical value $t \in \mathbb{C}$.

We identify $\mathbb{C}$ with $\mathbb{R}^{2}$ and write $f=P+i Q$, where $P$ and $Q$ are respectively the real and imaginary parts of $f$. We equip $\mathbb{C}^{n}$, when identified with $\mathbb{R}^{2 n}$, with the usual Euclidean structure. Then $\|\operatorname{grad} f\|=|\nabla P|=$ $|\nabla Q|$.

Assume now that $\varrho_{c}<1$. Let $c_{0}$ be a typical value of $f$ such that the real line $L \subset \mathbb{R}^{2}$ through $c$ and $c_{0}$ passes through no other generalised critical 
value, that is, $L \cap K(f)=\{c\}$. Let $V_{L}=f^{-1}(L)$. This is a smooth real algebraic hypersurface of $\mathbb{R}^{2 n}$. Let $f_{L}$ be the restriction of $f$ to $V_{L}$. The function $f_{L}$ is thus a smooth semialgebraic function, so $K_{\infty}\left(f_{L}\right)$ is finite. By definition, $f_{L}$ is a submersion.

We endow $V_{L}$ with the Riemannian structure induced by the Euclidean structure of $\mathbb{R}^{2 n}$ and denote by $\nabla_{V_{L}}$ the gradient with respect to the metric induced on $V_{L}$. After a rotation in $\mathbb{C}=\mathbb{R}^{2}$, we can assume, writing $c=a+i b$, that the line $L$ is $\{y=b\}$, where $(x, y)$ is a system of coordinates of $\mathbb{R}^{2}$. Then obviously $V_{L}=Q^{-1}(b)$, and since $\nabla P$ and $\nabla Q$ are orthogonal vector fields in $\mathbb{R}^{2 n}$, we deduce that

$$
\nabla_{V_{L}} f_{L}=\nabla_{V_{L}}\left(P_{\mid V_{L}}\right)=(\nabla P)_{\mid V_{L}}, \quad \text { so } \quad\|\operatorname{grad} f(v)\|=\left|\nabla_{V_{L}} f_{L}(v)\right|, \forall v \in V_{L} \text {. }
$$

From Remark 3.3, the Kurdyka-- -ojasiewicz exponent of $f_{L}$ at infinity for $c$, denoted by $\varrho_{c}^{L}$, is well defined, and we have just proved that $\varrho_{c}^{L} \geq \varrho_{c}$. If $\varrho_{c}<1$, by Remark 3.6, the fibre $f_{L}^{-1}(c)$ is diffeomorphic to $f_{L}^{-1}\left(c_{0}\right)$. Thus $f^{-1}(c)$ is also diffeomorphic to $f^{-1}\left(c_{0}\right)$, which is impossible since $c$ is a bifurcation value. Hence $\varrho_{c}=1$.

5. Examples. In this section we produce some examples that illustrate the results stated before. All the polynomials presented below have one asymptotic critical value. Each example describes a different phenomenon.

EXAMPLE 5.1 (Broughton example). Let

$$
f(x, y)=y(x y-1) \text {. }
$$

We immediately find that $f$ has no critical point. The set $\left\{\partial_{y} f=0\right\}$ is the algebraic curve $\{2 x y-1=0\}$ and $f(x, 1 / 2 x) \rightarrow 0$ as $x \rightarrow \infty$, and $0 \in K_{\infty}(f)$. Estimating the function $|x| \cdot|\nabla f(x)|$ along this half-branch at infinity shows that the Kurdyka--Łojasiewicz exponent at infinity $\varrho_{0}$ is equal to 1.

Since 0 is the only generalised critical value, we deduce $B(f)=K_{\infty}(f)=\{0\}$.

Denoting by $\phi_{t}$ the embedding of Theorem 2.3, we observe that the complement of $\phi_{t}\left(f^{-1}(0)\right)$ in $f^{-1}(t)$ is non-empty for all $t>0$. Taking $-f$ instead of $f$, we have a similar result for all $t<0$.

In this example the following is true: in the upper half-plane, there is a unique integral curve of $\nabla f$ which is of infinite length at 0.

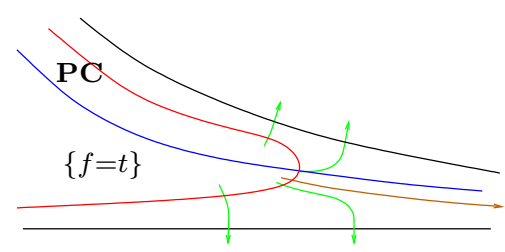

Fig. 1. Phase portrait of $\nabla f$ 
EXAMPLE 5.2 (King, Tibăr \& Zaharia example). Let

$$
g(x, y)=-y\left(2 x^{2} y^{2}-9 x y+12\right) .
$$

This function induces a smooth locally trivial fibration (see [TZ, Proposition 2.6]).

We obtain $K(g)=K_{\infty}(g)=\{0\}$, and $B(g)$ is empty. Any level $\left\{-y\left(2 x^{2} y^{2}\right.\right.$ $-9 x y+12)=t\}$ is homeomorphic to a line.

We compactify $\mathbb{R}^{2}$ to $\mathbb{R}^{2}$, with coordinates $[x: y: z]$. The point $[1: 0: 0]$ is the unique point at infinity of each fibre of $g$, and $\left\{\partial_{y} g=0\right\}$ is the union of the algebraic curves $\mathbf{P C}_{1}:=\{x y-1=0\}$ and $\mathbf{P C}_{2}:=\{x y-2=0\}$. As in the Broughton example, estimating the function $|x| \cdot|\nabla f(x)|$ along $\mathbf{P C}_{1}$ (or $\mathbf{P C}_{2}$ ) shows that the Kurdyka-Lojasiewicz exponent at infinity for 0 is equal to 1.

For this function, there are infinitely many trajectories of infinite length at 0 , meaning the trivialisation by the gradient near the value 0 is impossible.

Let $\mathbf{P C}_{v}=\{4 x y-9=0\}$ be the polar curve in the vertical direction. These three polar curves give enough information on the dynamics at infinity of the gradient field. A trajectory has at most one intersection point with each of the polar curves $\mathbf{P C}_{*}$ (with $*=1,2, v$ ). The phase portrait of $\nabla g$ is organised around two special integral curves (one between the $x$-axis and $\mathbf{P C}_{1}$, the other one between $\mathbf{P} \mathbf{C}_{2}$ and $\mathbf{P C}$ ), which actually are branching points of the space of leaves of the foliation by $\nabla g$. For any level $t>0$, the same kind of phenomenon occurs because of the symmetry of $g$.

A quick study of the signs of $\partial_{x} g$ and $\partial_{y} g$, and the study of the inflection points of the trajectories give enough information to draw the phase portrait of Fig. 2.

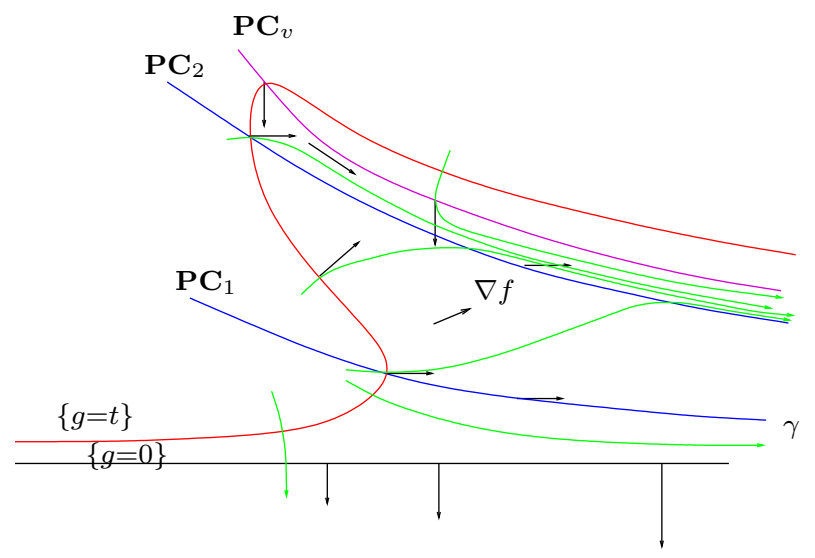

Fig. 2. Phase portrait of $\nabla g$ 
EXAMPLE 5.3 (Parusiński example). Let

$$
h(x, y)=y^{11}+\left(1+\left(1+x^{2}\right) y\right)^{3} .
$$

Each fibre of this function is homeomorphic to a line. Hence, by [TZ, Proposition 2.6], $h$ is a locally trivial fibration. On the curve $\mathbf{P C}_{v}:=\left\{\partial_{x} h=0\right\}=$ $\left\{1+\left(1+x^{2}\right) y=0\right\}$, we see that 0 belongs to $K_{\infty}(h)$. Moreover we find that $K(h)=K_{\infty}(h)=\{0\}$.

We compactify $\mathbb{R}^{2}$ to $\mathbb{R P}^{2}$, with coordinates $[x: y: z]$. Each fibre of $h$ admits $[1: 0: 0]$ as a unique point at infinity.

In this example, the gradient field realises the trivialisation.

The gradient vector field of $h$ is given by

$$
\nabla h(x, y)=6 x y\left(1+y+x^{2} y\right)^{2} \frac{\partial}{\partial x}+\left(11 y^{10}+3\left(1+x^{2}\right)\left(1+y+x^{2} y\right)^{2}\right) \frac{\partial}{\partial y} .
$$

Note that any level $h^{-1}(t)$, with $|t| \ll 1$, is actually the graph of some function $x_{t}$ of $y$, and we have

with $k(t)<0$.

$$
x_{t}(y)=\sqrt{\frac{t^{1 / 3}-1-y}{y}}+\text { h.o.t. } \simeq \frac{k(t)}{y^{1 / 2}},
$$

Let $\varrho_{0}$ be the Kurdyka-Lojasiewicz exponent at infinity for 0 . Let $G$ be any semialgebraic curve along which $h$ is negative and tends to 0 . The curve $G$ is the graph of a function, say $\kappa$, of the variable $x$. Thus we must have $\kappa(x) \sim-x^{\nu}$ for a rational number $\nu<1$. We assume $x \gg 1$.

If $\nu \neq-2$, it is easy to verify that

$$
|(x, \kappa(x))| \cdot|\nabla h(x, \kappa(x))| \geq x^{3} .
$$

Thus the Kurdyka-Łojasiewicz exponent along any such curve is nonpositive.

Assume $\nu=-2$. Then we deduce $\kappa(x) \simeq-x^{-2}$. So there exists $\eta>1$ such that $\partial_{x} h(x, \kappa(x)) \sim x^{-\eta}$, thus $\partial_{y} h(x, \kappa(x)) \geq 3 x^{3-\eta}$, and so

$$
|(x, \kappa(x))| \cdot|\nabla h(x, \kappa(x))| \simeq x \partial_{y} h(x, \kappa(x)) .
$$

We can verify that there is a positive constant $C$ such that:

(1) if $\eta \geq 23$ then

$$
x \partial_{y} h(x, \kappa(x)) \geq C|h(x, \kappa(x))|^{19 / 22}
$$

(2) if $\eta \in] 47 / 3,23[$ then

$$
x \partial_{y} h(x, \kappa(x)) \geq C|f(x, \kappa(x))|^{(4-\eta) / 22} \geq C|h(x, \kappa(x))|^{19 / 22} ;
$$

(3) if $\eta \in] 1,47 / 3]$ then

$$
x \partial_{y} h(x, \kappa(x)) \geq C|f(x, \kappa(x))|^{(8-2 \eta) /(3-3 \eta)} \geq C|h(x, \kappa(x))|^{2 / 3} .
$$


Taking $\kappa(x):=-\left(1+x^{2}\right)^{-1}$, we can verify that along $y=\kappa(x)$,

$$
x \partial_{y} h(x, \kappa(x)) \sim|h(x, \kappa(x))|^{19 / 22},
$$

and thus $\varrho_{0}=19 / 22$. So the flow of $\nabla h /|\nabla h|^{2}$ realises the trivialisation.

Thanks. The authors would like to thank the geometry team of University of Savoie, especially K. Kurdyka, P. Orro, and S. Simon for many talks and encouragement. We also thank R. Moussu and A. Parusiński for fruitful discussions. We are grateful to the referee for helpful comments and remarks.

\section{References}

[Br] S. A. Broughton, On the topology of polynomial hypersurfaces, in: Proc. Sympos. Pure Math. 40, Part 1, Amer. Math. Soc., 1983, 165-178.

[D'A1] D. D'Acunto, Valeurs critiques asymptotiques d'une fonction définissable dans une structure o-minimale, Ann. Polon. Math. 75 (2000), 35-45.

[D'A2] - Sur la topologie des fibres d'une fonction définissable dans une structure ominimale, C. R. Acad. Sci. Paris Math. 337 (2003), 327-330.

[Ha] H. V. Ha, Nombres de Eojasiewicz et singularités à l'infini des polynômes de deux variables complexes, ibid. 311 (1990), 429-432.

[KMP] K. Kurdyka, T. Mostowski and A. Parusinski, Proof of the gradient conjecture of R. Thom, Ann. of Math. 152 (2000), 763-792.

[KOS] K. Kurdyka, P. Orro and S. Simon, Semialgebraic Sard theorem for generalized critical values, J. Differential Geom. 56 (2000), 67-92.

[Pa] A. Parusiński, On the bifurcation set of complex polynomial with isolated singularities at infinity, Compos. Math. 97 (1995), 369-384.

[Ph] F. Pham, La descente des cols par les onglets de Lefschetz, avec vues sur GaussManin, in: Systèmes différentiels et singularités (Luminy, 1983), Astérisque 130 (1983), 11-47.

[Th] R. Thom, Ensembles et morphismes stratifiés, Bull. Amer. Math. Soc. 75 (1969), 240-282.

[Ti1] M. Tibăr, On the monodromy fibration of polynomial functions with singularities at infinity, C. R. Acad. Sci. Paris Sér. I Math. 324 (1997), 1031-1035.

[Ti2] - Regularity at infinity of real and complex polynomial functions, in: Singularity Theory, B. Bruce and D. Mond (eds.), London Math. Soc. Lecture Note Ser. 263, Cambridge Univ. Press, 1999, 249-264.

[TZ] M. Tibăr and A. Zaharia, Asymptotic behaviour of families of real curves, Manuscripta Math. 99 (1999), 383-393.

[Ve] J.-L. Verdier, Stratifications de Whitney et théorème de Bertini-Sard, Invent. Math. 36 (1976), 295-312.

Dipartimento di Matematica

Università degli Studi di Pisa

Via Filippo Buonarotti 2

56127 Pisa, Italy

E-mail: dacunto@mail.dm.unipi.it ddacu@univ-savoie.fr
Department of Computer Science University of Bath Bath BA2 7AY, England, UK E-mail: cssvg@bath.ac.uk 